\title{
El profesional de la información y su capacitación permanente en un entorno signado por los cambios
}

\author{
Elsa Román \\ Ana Pioli \\ Escuela Universitaria de Bibliotecología \\ y Ciencias Afines (Montevideo, Uruguay)
}

\subsection{Resumen}

Reflexión sobre el futuro del profesional de la información y los retos que debe afrontar a finales del siglo XX. Se intenta demostrar que los cambios afectarán positivamente al estatus del bibliotecólogo, le añadirán nuevos roles a los ya existentes y plantearán desafíos en su formación, su función social y cultural y sus estrategias de integración regional. La formación, la investigación en el área de la información y un mayor vínculo interdisciplinario son premisas de las que emergerá el gestor de la información que el siglo XXI demanda.

Palabras clave: Centro de Documentación. Modernización. Relaciones. Laborales. Uruguay.

\section{Abstract}

Reflections on the future of the information professional at the end of the 20 th century. While putting pressure in permanent and formal education, perception of the social and cultural role and regional integration strategies, current changes will affect positively the status of the librarian and new roles will emerge. Education and training, research and interdisciplinary interaction are the premises from which the new information manager of the 21 st century is to be born.

Keywords: Documentation centres. Modernisation. Labour relations. Uruguay.

\section{Introducción}

Este entorno de fin de siglo, nos ubica en un mundo en "reconstrucción", las economías transitan de una sociedad altamente industrializada, a otra que algunos autores han denominado "sociedad de información", "era post-industrial", etc. 
Más allá de las designaciones se torna evidencia que no existe una teoría preestablecida, que la rutina no tiene lugar, que los hechos nos asaltan, los paradigmas se caen, se rompió el mundo bipolar y la globalización nos coloca frente a un fenómeno internacional para el cual no existe una doctrina suficiente, las teorías herméticas no han sido sustituidas por nuevos paradigmas y el camino es incierto.

Se percibe el nacimiento de una nueva era, en formación, en tránsito, en borrador, que en algunos países está adquiriendo forma más rápidamente que en otros. Ante estos retos de la historia, el tema central, sin lugar a dudas, es el empleo y las nuevas formas de relacionamiento entre los actores sociales involucrados (trabajadores-empresarios-estado).

Numerosos aspectos de la vida económica, cultural y social emergen: métodos y relaciones laborales, condiciones de trabajo, niveles de empleo, organización de las empresas, organización sindical, objetivos de la educación y la formación, y el modo en que las personas se comunican entre sí.

El sector información aparece como un sub-sistema con una estructura y características propias que necesariamente deberá ajustarse al medio con un enfoque sistémico para lograr niveles de excelencia en un entorno de innovación y consenso.

La competitividad es un indicador para los productos de información. Esta hipótesis nos lleva a reconsiderar las prácticas de la organización del trabajo. Los tiempos y ritmos de trabajo ya no están más determinados por las organizaciones en su interior. Los condicionantes les vienen dados desde fuera.

Las nuevas estrategias de cooperación, y una arquitectura organizacional diferente, requieren un alto grado de especialización en el trabajo, lo que provoca la necesidad de gerenciar la información y la formación.

La pregunta central para los profesionales de la información, entre ellos el bibliotecólogo, quien nos ocupa en este trabajo, es acerca del status que tendrán en dicho entorno y qué modelos emergen como posicionamientos innovadores cuando el desarrollo de las tecnologías de la información y la comunicación están transformando profundamente numerosos aspectos de la vida económica, cultural y social.

Este ambiente caracterizado por el crecimiento e importancia de la industria de la información y del conocimiento, ha impactado fuertemente a las organizaciones tanto públicas como privadas que, ante las nuevas demandas surgidas de un entorno donde lo único permanente es el cambio, deben perseguir una readecuación permanente. 
Se hace impostergable el contextualizar dichos cambios porque transforman radicalmente el valor de la información y afectan a nuestros estilos de vida.

Esta nueva realidad hace que aparezcan como necesarios los cambios en los currícula de la profesión del bibliotecólogo con la finalidad de lograr una reinserción activa en la región. Será necesario un reposicionamiento del profesional de la información, y un replanteamiento de su formación en este proceso de integración regional que nos permita adoptar estrategias de acción conjunta para mejorar el status en las respectivas comunidades de la región.

\section{El contexto de los cambios y el fenomeno informacional}

El propósito de este artículo es delinear algunas tendencias de estos cambios.

¿Cómo han ido planteando otros países la problemática del fenómeno informacional a la luz de los cambios anteriormente mencionados?

Europa comienza su proceso de integración por razones políticas: aspiraba transformarse en la tercera potencia en el mundo, después de la $2^{\mathrm{a}}$ Guerra Mundial, mundo marcado por el bi-polarismo: EEUU-URSS.

El inicio de estas negociaciones se ubica en dos tratados: el de París firmado en 1950 y el de Roma en 1951. Cuarenta años después Europa ha logrado sus objetivos y ha desarrollado programas que priorizan distintas políticas.

Al mirar hoy a la Comunidad Europea visualizamos un conjunto de programas que atienden temas educativos: PETRAS, ERASMUS, COMET, FORCE, EUROTECNET, REDNARIC.

En el área de la información el G7 ha desarrollado también actividades dirigidas al sector. El proyecto Information Society Project Office, ISPO, es parte del plan de la Comisión para visualizar y planificar la manera en que Europa se inserta en la sociedad de información.

Este proyecto nace en el año 1994 para apoyar, promover y orientar las acciones públicas y privadas con relación a la sociedad de información. "Los europeos podrán aspirar a nuevas oportunidades de trabajo, nuevos servicios y nuevos mercados, en el despertar de la Sociedad de la Información" (Bangemann, 1994).

Se cree que esta afirmación es particularmente cierta en el campo de nuestra profesión, que tiene ante sí un muy amplio y variado abanico de nuevos mercados en los cuales desarrollar su actividad.

Algunas preguntas aparecen como claves en estos nuevos escenarios:

- ¿Qué destrezas y habilidades serán requeridas por este nuevo profesional?

- ¿Qué institución de formación se quiere?

Scire. 4 : 2 (jul.-dic. 1998) 23-33. 
- ¿En qué profesional se piensa?

No es la intención de este artículo, dar respuestas definitivas a una problemática que requiere de la reflexión colectiva, de una puesta a punto, tanto en relación con la concepción como a la conceptualización del status profesional requerido, sino aportar elementos para una discusión, que en nuestro medio, es impostergable.

Nuevas ocupaciones se crean; nuevas oportunidades se vislumbran; nuevos ámbitos de acción aparecen y nuevas habilidades son demandadas. ¿Cómo fueron respondiendo las distintas instituciones de formación profesional a esta realidad?

En la década del 80 la mayoría de las escuelas de bibliotecología y ciencia de información de Gran Bretaña cambiaban sus nombres destacando la componente información. Una década más tarde, en los Estados Unidos, se observaba una tendencia semejante y es así como la Escuela de Estudios de Biblioteca e Información de la Universidad de California, Berkeley cambia su nombre por la de Escuela de Estudios de Información.

Tanto en Estados Unidos como en Gran Bretaña, ya hacía mucho tiempo, dos de las mejores escuelas, Syracusa y Sheffield, habían decidido que la expresión más apropiada para definir intereses heterogéneos — profesionales y académicos- era "estudios de información". Se comprendían cabalmente las profundas implicancias pedagógicas y mercadológicas de la revolución de la información y las telecomunicaciones y por lo tanto se ajustaban continuamente sus ofertas de programas y sus líneas de investigación.

Una gran paradoja que se comenzó a dar en este panorama educacional norteamericano es que en la medida que la sociedad de información se desarrollaba, un número creciente de escuelas de bibliotecología estaba siendo eliminado. Desde 1978, por lo menos 15 escuelas norteamericanas de bibliotecología y ciencia de la información desaparecieron y otras tantas han visto peligrar su futuro. Cabría preguntarse por qué sucedió esto y por qué se asumen posturas defensivas cuando las oportunidades para el crecimiento y renovación en el contexto de la sociedad de información se muestran tan evidentes.

A partir de la década del 80 comienzan a percibirse en América Latina, nuevas actitudes, nuevos términos como "mercado de trabajo emergente", mercado de trabajo invisible", etc., asimilado a ritmos diferentes en los distintos países.

El MERCOSUR, proceso de Integración Regional del cual Uruguay forma parte, ha generado en el ámbito académico instancias de intercambio y discusión acerca de la formación del profesional de la Información en este nuevo contexto de mercados emergentes. Diferentes foros y simposios han colocado en primer 
plano la necesidad de adecuación de dichos profesionales a las necesidades del mercado de trabajo (Castilla y Pérez, 1995).

En nuestro país - Uruguay - la Escuela Universitaria de Bibliotecología y Ciencias Afines está efectuando un esfuerzo significativo en pos de los cambios: en el marco del programa de Educación Permanente, año tras año se organizan cursos de posgrado con participación de docentes nacionales y extranjeros; y se implementa un Programa de Formación y Perfeccionamiento para los docentes de la Escuela.

Asimismo, los estudiantes que cursan el Seminario Taller en el cuarto año de la carrera, encaran temáticas interesantes para sus proyectos finales que analizan los grandes cambios que afectan a nuestra profesión, entre los que se destacan temas tales como: la gestión de la información en la empresa; mercado tradicional y emergente; ejercicio liberal de la profesión; evaluación del plan de estudios, etc.

Dentro de la complejidad e incertidumbres de este futuro, - futuro que todos los profesionales del área de la información, ya sean éstos: bibliotecólogos, archivólogos, documentalistas, periodistas, editores, comunicadores sociales, informáticos, etc., tenemos la responsabilidad de crear con imaginación e inventiva-, algunas tendencias futuras se pueden delinear.

En algunos estudios se ha abordado que las nuevas tecnologías de información y comunicación, planeamient y economía son asignaturas que aparecen como deficientes en la formación del bibliotecólogo.

Se menciona con bastante claridad la necesidad de la emergencia de un profesional con fuerte formación en gestión de la información. Este profesional del próximo milenio combinará habilidades tecnológicas y capacidades de analizar políticas con una fuerte orientación hacia el cliente.

Estamos hablando de un profesional con capacidad para posicionarse o reinsertarse en diferentes ambientes organizacionales que, nos agrade o no, se encuentran en un acelerado proceso de transformación.

Muy recientemente el grupo de trabajo (FID-IFLA, 1998) recomendó que las Escuelas de Bibliotecología y estudios de Información de la región reforzaran la enseñanza de modernas técnicas de gestión así como que incentivaran la investigación en éste área y que estimularan el desarrollo de capacidades de liderazgo en los estudiantes.

En primer lugar parece oportuno definir el alcance de términos 'gestor' y 'gestión de información'. Gestor de información se puede definir como aquel que cumple un rol en la "Inteligencia de la información”, es decir, aquél que le agre- 
ga información a la información para transformarla casi en conocimiento (CEPAL-CLADES, 1997).

Por otra parte, (Orna, 1994), define la gestión de la información como "el conjunto de instancias responsables de la definición de políticas y acciones en la organización con relación a: la forma como la información se adquiere, registra y guarda, cómo la información se usa y se comunica; la manera cómo las personas que manejan la información aplican sus habilidades y cooperan entre ellas; la efectividad con que las actividades relacionadas con la información contribuyen al logro de los objetivos de las organizaciones y los individuos; la forma cómo se usan las tecnologías de la información en todas las actividades; los costos y beneficios que conllevan las actividades de información".

Por último, señalaremos a (Olaisen, 1995) quien afirma: "La gestión de recursos de información, Information Resource Management, es la gestión integrada de la información (interna y externa) y de las tecnologías de la informació, aplicadas a las áreas estratégicas y a los factores críticos de competitividad de la organización. La GRI trata de enlazar la efectividad de la gestión de la empresa con la adquisición y utilización de la información. Se basa en la premisa de que la toma de decisiones y el pensamiento estratégico no pueden ser desligados del acto de proceso de información."

Los cambios que se están procesando en la organización del trabajo - pasaje de una organización taylorista, vertical, a otra más horizontal, en red- exigen del gestor una visión global de los componentes, recursos y procesos que el profesional de la información deberá tomar en cuenta para aggiornar su labor.

\section{Rol de las bibliotecas}

Las bibliotecas están viviendo una transición: de funciones de conservación pasaron a las de difusión de la información y de éstas a las bibliotecas o unidades de información como lugar de acceso a la información independientemente del lugar donde ésta se encuentre.

Así, la formación del profesional de la información que demandan los nuevos tiempos ha ido y va cambiando. Hace relativamente pocos años, la erudición en las actividades de procesamiento de la documentación (catalogación, clasificación) era una de las cualidades más apreciadas; en el futuro, es posible incluso que estas tareas vengan incluidas en los documentos que se adquieren o que se apliquen los mecanismos llamados de outsourcing: contratación de una empresa externa para su ejecución.

Al primar cada vez más la calidad de los servicios de acceso a la información, es su gestión y la de los servicios al público en general una de las cualidades más necesarias. El perfil del profesional de la información deberá incluir 
conocimientos en técnicas de estudio de mercado, técnicas de calidad, estudios de usuarios, técnicas de muestreos y encuestas, marketing, planeamiento estratégico y consultoría, entre otros.

Existe una demanda de gerentes de recursos de información, especialistas que no se limiten a identificar solamente dichos recursos sino que además se ocupen del análisis y síntesis de la información de forma tal que puedan producir servicios y productos con un alto valor agregado, dirigidos a una clientela específica. Profesionales capaces de gestionar recursos humanos, tecnológicos, materiales y de información.

Las funciones relativas a la venta de servicios de información y "reempaquetamiento de información”, vale decir el análisis y síntesis de la información para alcanzar las necesidades de una clientela específica deberían estar en manos de un information counselor con conocimiento en estas tareas de comunicación y técnicas sociales.

El otro aspecto clave para avanzar en la búsqueda de respuestas desde el sector información, es la necesidad de crear áreas de especialización según prioridades nacionales de desarrollo:

- Información - sector productivo

- Información - educación

- Información - investigación.

Finalmente, el motor que alimentará todo este proceso es la investigación. Investigación que deberá redimensionarse, fortalecerse, ser generadora de conocimiento teórico.

Si hacemos un rápido repaso de los principales pensadores del siglo XX con relación a la bibliotecología, se puede comprobar que hoy por hoy se continúa enfatizando la importancia de estructurar teóricamente a esta disciplina sorteando obstáculos tales como: las afirmaciones que se producen con frecuencia acerca de que la actividad bibliotecaria no posee carácter científico, puesto que los trabajos producidos, por ejemplo, en el área de la clasificación y catalogación, fueron motivados por problemas inmediatos y prácticos en bibliotecas específicas; o el problema concerniente a la falta de continuidad en el desarrollo de una teoría, integrando las dificultades que puedan derivarse de sus aplicaciones inmediatas o de la generalización de su uso que se requiere para consolidarse como teoría válida.

A principios del siglo XX los argumentos a favor de la investigación teórica no estaban enfocados tanto a la potenciación interna de la bibliotecología como a la formación del bibliotecario para que éste adquiriera una conciencia clara de la función social y educativa que poseía la biblioteca. El contexto de la biblioteca

Scire. 4 : 2 (jul.-dic. 1998) 23-33. 
pública es el horizonte de reflexiones de la época, cuyos pensadores más representativos son: J. R.Wilson, C. Williamson, y Greeword.

En las décadas de los años 60 y 70, los pensadores P. N.Ennis, H. Goldstein y D. G. Shaffer coincidían en términos generales, en la necesidad de un cuerpo teórico para la disciplina que permitiera la integración, fortalecimiento y continuidad de los conocimientos producidos.

Por su parte, (Shera, 1973) en su obra, "Fundamentos de la educación para bibliotecólogos", estudia desde la perspectiva filosófica la bibliotecología, la actividad bibliotecaria y la educación con el propósito de integrar estas reflexiones bajo un fundamento epistemológico que le permitiera postular la tesis de la bibliotecología como ciencia social y a sus reflexiones como epistemología social.

Cada campo científico es definido por los problemas que él identifica y elige para su estudio y solución. Por lo tanto, el objeto de estudio de la bibliotecología sigue siendo, en nuestra opinión, merecedor de análisis por parte de los actuales profesionales y significa un reto para realizar una tarea que es demandada por la comunidad de bibliotecólogos con el propósito de lograr un reposicionamiento tratando de visualizar en el entorno oportunidades y no amenazas.

\section{El trabajo interdisciplinario}

Son múltiples los estudios que el profesional de la información puede abordar conjuntamente con otras disciplinas:

\subsection{Investigación científica}

- Estudios bibliométricos aplicados a la evaluación de la ciencia y la tecnología.

- Medición de la producción científica de un país en áreas especializadas.

- Tamaño, crecimiento y distribución de la bibliografía científica con el objeto de mejorar las actividades de información y documentación.

- Análisis de los procesos de generación, propagación y uso de la literatura científica para conocer los mecanismos de la investigación considerada como actividad social.

\subsection{Sociología - Ciencias de la Comunicación}

- Estudios de las conductas informativas de las comunidades científicas.

- Modelos del pensamiento del usuario final de la información en el marco del desarrollo de las telecomunicaciones. 


\subsection{Lingüística}

- Lenguajes naturales de indización.

- Nueva organización del conocimiento y adecuación de los lenguajes de recuperación.

\section{Ingeniería}

- Redes y sistemas de información

- Interfaces de comunicación usuario-sistema

- Estudios de servicios de información en la red.

\section{Información-sector productivo}

- Incidencia del desarrollo de las telecomunicaciones en el posicionamiento competitivo de las empresas y el rol de la información como recurso para el logro de esta meta.

- Estudios acerca del comportamiento de los distintos actores sociales (empresarios, trabajadores, estado) frente a la información como recurso en el proceso de globalización.

\section{Conclusiones}

Sin duda, los retos que esta nueva era pone ante nosotros son múltiples.

El saber visualizar esta situación como una oportunidad para el desarrollo de nuestra profesión es un desafío que nos convoca a todos: docentes, egresados, estudiantes y usuarios finales de la información.

Estamos viviendo una cultura innovadora, cualquier innovación deberá tener en cuenta las necesidades del medio socioeconómico y los recursos implicados en el emprendimiento.

Este nuevo profesional del que hablamos, con una marcada formación en gestión de recursos, deberá monitorear permanentemente este ambiente de cambios para brindar respuestas adecuadas a un mercado en el que los competidores - en relación al trabajo con la información- se multiplican.

En primer lugar este profesional deberá conocer con mucha precisión ese mercado: cómo se han adaptado las unidades de información a los nuevo desa fíos, cuáles son las unidades punteras, qué procesos han realizado para alcanzar sus metas, qué reposicionamiento han logrado y cómo se formaron los gestores de los cambios, a qué mercado va dirigido su conocimiento y su técnica, qué propuestas han efectuado en su campo de trabajo, y cómo se logra medir el impacto económico de los proyectos que emprenden.

Estos años de desarrollo de las profesiones de bibliotecólogo y archivólogo, especialmente en los países de América Latina, han mostrado a un profesional 
más empírico en su pensamiento que científico. De esta manera, los bibliotecólogos se ven esenciales en el trabajo de colaboración en investigaciones de otros campos del conocimiento, pero mantienen una actitud de desconfianza $-\mathrm{o}$ de pasivida-), priorizando investigaciones aplicadas y no de desarrollo hacia la investigación en su propio campo.

¿Cómo lograremos liderar entonces proyectos de investigación e innovación con transferencia efectiva sin estar preparados en técnicas de gestión, de manejo de recursos, de análisis de mercado, técnicas de estadística descriptiva e inferencial que componen el lenguaje de la investigación científica?

Creemos que la enseñanza mejor inspirada es la más cercana a la investigación. Por ello, se debe fomentar el espíritu científico, estimular desde el primer grado y a lo largo de toda la carrera, la investigación, la formulación de proyectos de investigación o de innovación.

$\mathrm{Si}$ acentuamos nuestro esfuerzo en este sentido, estaremos coadyuvando a formar un gestor siempre atento a las opiniones de los clientes y las demandas del entorno, anticipándose en innovaciones tecnológicas, ofreciendo nuevos productos y servicios de información, discutiendo y negociando con autoridades los presupuestos, presentando informes, etc. En otras palabras, un profesional responsable de un servicio o área de información que se convierte en el motor de la organización en la tarea de ofrecer ese dato, información o conocimiento que posibilitará la resolución del problema y la toma de decisiones en el momento y tiempo preciso.

\section{Bibliografía}

Agada, Johan (1996). Information professionals in a globally networked society : an agenda for social skills. // FID News Bulletin (1996)

Alba, Luis ; Gazitúa, José Miguel ; Cubillo, Julio (1997). Tres enfoques sobre el nuevo gestor de la información. // Información y Desarrollo. 8 (Febr. 1997).

Bangemann, Martín (1994). Information Society Project Office, Europe and the global information society : Recommendation to the European Council, Bruselas, 26 de mayo. URL: <http://www.ispo.cec.be> [consulta: abril 1997].

Bangemann, Martín (1997). A new world order for global comunicatons : the need for an international charter. URL: <http:www.ispo.cec.be/infosoc/promo/speech/ geneva.html> [Consulta: mayo, 1998].

Bettinsoli Guise, Nunzia ; [et al] (1997). Preparing librarians to meet the challenges of today's health care environment . // Journal of the American Medical Informatics Associatonn . $4: 1$ (jan-feb. 1997).

Castilla, María Teresa ; Perez, María Cristina (1995). Recursos Humanos para la industria de la información : enfoque del Uruguay en la perspectiva del Mercosur. // Anais do Simpósio de Biblioteconomia e Cièncias da Informação do Mercosul e XII Jornada 
Sul-Rio Grandense de Biblioteconomia e Documentação. Río Grande : FURG/ARB/ADIRU, 1995.

Coriat, Benjamin (1992). Pensar al revés : trabajo y organización en la empresa japonesa. España : Siglo XXI, 1992.

Cornellà, Alfons (1994). Los recursos de información : ventaja competitiva de las empresas. Madrid : McGraw-Hill, 1994.

Cronin, Blaise (1993). ¿Profissionnalização ou proletarização da atividade informacionnal?. // R. Esc. Bibliotecon. UFMG-BH. 22 : 1 (Jan-jun. 1993) 38-65.

FID/IFLA : Grupo participante (1998). Conclusions and recomendations. Valparaiso : s. e. 3 p. (Workshop on Management And Use of Human Resources in Library and Information Work in Latin America, Valparaiso, 6-9 abril, 1998).

Houser, Lloyd J. ; Lazorick, Gerald J. (1978). Introducing a significant statistics component into a library science research methods course. // Jour. Educ. Librarinaship. 18 : 3 (1978) 175.

Jiménez, Miguel. (1997) El futuro de los bibliotecarios. // Arbor. (Mayo-junio, 1997) 617618.

Leirado, Inés Isabel (1996). Revalorización de la educación como palanca para lograr una reinserción activa de los países del Mercosur en el sistema estratificado internacional. Ponencia presentada al: II Congreso Latino-Americano de Sociología del Trabajo. Aguas de Lindoia, San Pablo, Brasil, 5 de diciembre 1996.

Olaisen, J. (1995). Preface : Information and knowledge and organizational learning. // Svensk Biblioteks Forsknings. 14 (1995) 5.

Orna, Elizabeth (1994). Taking advantage of developments in the world information services and managing information. // Aslib Proceedings. 46 : 2 (February, 1994).

Rifkin, Jeremy (1996). El fin del trabajo : nuevas tecnologías contra puestos de trabajo, el nacimiento de una nueva era. Barcelona : Paidós, 1996.

Sanguinetti, Julio María (1997). Globalización, liberalización hemisférica e integración regional. SELA : Caracas. Conferencia ofrecida con motivo de su visita oficial a la sede de la Secretaría permanente del SELA, 20 de mayo de 1997.

SELA (1996). FORO : Impacto del euro en las relaciones económicas entre la UE y América Latina y el Caribe. Caracas, Venezuela 29 de octubre de 1996.

Shera, Jesse (1973). Towards a Theory of Librarianship and Information Science. // Ciencia da Informacao. 2 : 2 (1973) 87-97.

Trein, Franklin. (1998). Sociedad de la Información : experiencia europea. // $11^{\circ}$ Encuentro Latinoamericano de Informática e Industrias de la Información, ELAII, San José, 27-28 abril 1998 / con el auspicio de la Fundación Fontaina Minelli.

Williamson, C.C. (1931) The place of research in library service. // Library Quarterly. 1 : 1 (1931) 1-17.

Scire. 4 : 2 (jul.-dic. 1998) 23-33. 